\title{
MODELOS PARENTALES EN EL SURESTE DE MÉXICO, EL CASO DE LA ZONA DE LA CHONTALPA EN TABASCO, MÉXICO.
}

\author{
Dra. Juana Marín Turriza, \\ Dr. Euler Ferrer Córdova
}

\begin{abstract}
This paper aims to present a study of parental models in fathers and mothers, considering this institution as the space where economic, politic and social transformations produced by the social dynamics are reflected in several ways. The concept of parental models is understood as the set of norms and rules that regulate behaviors (breeding patterns), model of communications based on the time of coexistence and the ways tos how affection (links of attachment), and the promotion of guidelines linked to success (success patterns), aspects explored with the Atribucional Questionnaire on Parental Models (Martìnez et al., 2014), applied to 1900 families from the five municipalities that make up the Chontalpa region of the State of Tabasco. The study reveals that parents use negotiating and rigid breeding patterns, warm and close ties of attachment and patterns success counselors and promoters.
\end{abstract}

Keyword: parental models, breeding patterns, modernity, family adaptation.

*En este escrito la palabra padres hace alusión a las dos figuras parentales (padres y madres).

\section{Introducción}

El grupo familiar se articula a partir de las características que le confiere el contexto social, funcionando como un juego dialéctico que cambia a ambos grupos, es así que la familia depende del grupo social, el momento cultural e histórico que le rodea (Caballero y Castillo, 2003), de tal manera que se constituye y se transforma socialmente; en la composición de sus miembros, la cultura y el rol de los mismos, así como la manera en que se enfrenta a la modernidad, un ejemplo de estas adaptaciones es la entrada de ingreso proporcionado por más de un integrante, modificando con esto la forma en que se lleva la socialización de los hijos (González, 2011) y las 
expectativas respecto a la calidad de vida que ofrece la globalización.

Cadenas y Urquiza (2016) retoman a Luhmann, al señalar el funcionamiento diferenciado del sistema familiar a pesar de los cambios tan rápidos y drásticos que sufre, pues el individuo moderno, o no, necesita atribuirse señas de identidad específicas, una relación social y un vínculo parental. Los cambios sociales a los que ha estado expuesto este núcleo social dejan de lado a la familia tradicional, ahora puede ser llamada de formas variadas y poco diferenciadas; moderna, nuclear, que delega, que comparte, etc., apareciendo el abandono, las compensaciones y gratificaciones que remplazan la función parental ante la competencia económica (Rovira, Bernatzky y Amores, 2009).

La familia atomística como la llaman Rovira et al. (2009) está entonces ante una crisis al intentar seguir cumpliendo su función formadora, pero ante las demandas de éxito privilegiadas en los adultos, la marca o mentalidad familiar está manifiesta por el valor que se atribuye al éxito económico y las imágenes construidas socialmente. En este mismo sentido Caballero y Castillo (2003) señalan la desorganización familiar y el desequilibrio en su estructura de poder como uno de los factores a nivel microsocial que favorecen la adopción de conductas violentas o de desadaptación familiar.

Ante los cambios de la modernidad a los que responde la inquietud por la familia, también se hace necesario considerar a aquellas familias con lengua, cultura y memoria histórica diferente, y la forma en que responden o se adaptan a ellos (Rovira et al., 2009), un ejemplo de esto es la permeabilidad de la tendencia televisiva, que marca más prácticas y pautas de crianza que los mismos padres o cuidadores alcanzan a efectuar, con horarios televisivos que poco discriminan a su público y la falta de acompañamiento a los niños en esta actividad (Varela, Chinchilla y Murad, 2015), todo esto supone un mayor de grado de incertidumbre e inestabilidad como costo de su mantenimiento frente a las profundas transformaciones (Cadenas y Urquiza, 2016). Esta preocupación por la familia y la labor de crianza puede ser distinguida desde diferentes ámbitos.

- $\quad \mathrm{El}$

Contexto

sociocultural: donde la relación familiar se ve afectada por la crisis económica, el 
abuso de drogas, el maltrato psicológico, el maltrato físico y las separaciones (Garcés y Palacio, 2010), un ejemplo de ello es la migración de los padres que obliga a re-establecer a los hijos en nuevos lugares físicos y emocionales con un sustituto parental (López, 2012).

\section{- La Influencia}

generacional y de la familia extensa: donde desde la primera hasta la tercera generación en aspectos ético-afectivos se evidencian las prácticas de crianza guiadas por el régimen socioeconómico y el carácter de la relación familiar (funciones, jerarquías, espiritualidad, educación, etc.) que envisten las creencias sobre la crianza (García y Salazar, 2013).

\section{- La estructura familiar:} donde las familias nucleares favorecen la comunicación, por sobre aquellas familias monoparentales y extensas (Garcés y Palacio, 2010), no siendo esta característica definitoria, pues el poco apoyo percibido de la pareja, la no percepción de la importancia al establecer límites y ofrecer autonomía, contribuyen a prácticas severas en la práctica parental (Solís y Díaz, 2007).

- La estabilidad emocional y capacidades del cuidador: donde la salud mental de padres y responsables del cuidado (padecimiento de estrés, depresión, agresividad, etc.), afecta directamente el estilo de crianza y la relación, dada la reacción ante conductas y emociones (Cuervo, 2010).

- La Interacción padre-hijo: donde las diferencias en la relación filial son influidas por el sexo de los hijos, afectando la conducta e interacción respecto a cómo deben ser y como son con sus hijos (Solís y Días, 2007).

El trabajo y énfasis sobre la familia y la labor de crianza, se evidencia en los servicios y apoyos para su funcionamiento en la sociedad actual, provistos por el gobierno español, a fin de potenciar aquellos factores de protección emergidos de y para el núcleo familiar (Rodrigo, Máiquez y Martín, 2010), también se han identificado los modelos parentales en el contexto urbano (Martínez et al., 2014; Martínez, García y Collazo, 2017), así como las concepciones y ejes de racionalidad en torno a la familia, y las teorías asumidas respecto a ella y sus funciones de crianza (Martínez-Licona, Infante y Medina, 2016; Martínez, Rodríguez, Díaz y Reyes, 2018). 
Ante este estado de manifestaciones es conveniente explorar no solo lo que sucede en un modelo de familia ligada a núcleos sociales urbanos contemporáneos, si no conocer lo que está sucediendo con esta institución en zonas con características distintas, tanto geográficas como culturales, aspecto que cumple la zona Chontalpa del estado de Tabasco, compuesta por cinco municipios, cuya actividad económica y cultural se caracteriza por la intensa actividad petrolera, su producción de cacao, caña de azúcar, piña, etc., además de aprovechar sus zonas arqueológicas y playas para la actividad turística.

El objetivo principal de este estudio es explorar y describir las características de los modelos parentales en familias de la región Chontalpa, haciendo hincapié en los principios que dan soporte al modelo parental ejercido en la dinámica familiar.

\section{Objetivo}

El objetivo del estudio es presentar las características de los modelos parentales más frecuentes en la zona de la Chontalpa, haciendo énfasis en las pautas de crianza, los vínculos de apego y los patrones de éxito fomentados por los padres y madres del sureste de México.

\section{Materiales Y Métodos}

\section{Método}

Para la aplicación del instrumento fueron capacitados aproximadamente 50 alumnos de la Universidad de la Chontalpa. Se describen los resultados a partir del Cuestionario de Modelos Parentales (Martínez et al., 2014), mismo que involucra el pensamiento atribucional (ligado a la acción), respecto a la dinámica familiar y las estrategias de crianza.

\section{Participantes}

1905 familias de la región de la Chontalpa, pertenecientes a los cinco municipios que la conforman, con un promedio de 380 aplicaciones por municipio.

\section{Técnica de análisis}

Análisis de distribución de frecuencias, designando una categoría para cada factor del modelo parental, los datos fueron procesados para lograr indicadores sobre la caracterización de 
las pautas de crianza, los vínculos de apego y los patrones de éxito que las familias atribuyen a la propia práctica.

\section{Instrumento}

El instrumento desarrollado por Martínez et al. (2014), identifica el modelo parental a partir de 33 enunciados, organizados en 12 reactivos; explora las pautas de crianza, los vínculos de apego y los patrones de éxito implicados en el clima familiar. Es así como a través de los niveles funcionales del pensamiento (Martínez et al., 2014) se busca que las personas reconozcan versiones prototípicas

o representacionales del pensamiento, y asuman como propia (atribución) una versión ejemplificada en los enunciados contenidos en el instrumento.

El perfil del modelo parental se estructura en función de las normas y la disciplina (pautas de crianza), el tiempo compartido, el afecto y la comunicación (vínculos de apego), la promoción y el control del éxito (patrones de éxito), conceptualizándose de la siguiente manera:

Las pautas de crianza se identifican como rígidas (convivencia fundamentada en el orden, normas y limites rígidos, donde la obediencia y el respeto a la autoridad fundamentan la dinámica familiar), negociadoras (las normas y limites se manifiestan solo en función de las necesidades de los hijos o pretensión de los padres) e indulgentes (los hijos gozan de libertad y se conducen conforme a sus intereses e impulsos, por la independencia que se les otorga) (Martínez et al., 2014).

Los vínculos de apego se ligan directamente a las demostraciones de afecto; en su forma, momento y tiempo. De esta manera los padres pueden ser cálidos (el afecto es parte de la vida cotidiana y caracterizada por su calidez y cercanía), cercanos (el afecto se manifiesta espontánea y esporádicamente, aunque con tiempo y comunicación suficiente) y elementales (hay mínimas muestras de afecto y disposición de tiempo, con prácticamente nula comunicación) (Martínez et al., 2014).

Los patrones de éxito se refieren a la promoción y expectativas de éxito que son suscitadas en la familia de manera normativa y disciplinar (a través de control y evaluación de las conductas que conducen al éxito). Un patrón de éxito promotor contribuye a las 
decisiones de los hijos sustentadas en los intereses de los padres, un padre orientador a partir de los intereses de sus hijos ayuda a que construyan su patrón de éxito, y la condescendencia o emancipación (Martínez-Licona et al., 2016) se refiere a la aceptación o respeto del patrón asumido y decidido por los hijos (Martínez et al., 2014).

Cuando no se identifica un patrón consistente hacia algún tipo de pauta de crianza, vinculo de apego o patrón de éxito, implica que el modelo parental varía de acuerdo con las situaciones o incluso el estado emocional y no como fruto de la cultura familiar estable (Martínez et al., 2014), por lo que se les denomina "situacionales".

\section{Población}

\section{Tabla 1}

Edad de los padres y madres de la exploración

\begin{tabular}{|c|c|c|c|c|c|}
\hline Edad & $\mathrm{C}$ & Por & Nive & $\mathrm{C}$ & Po \\
\hline de los padres & antidad & centaje & $\begin{array}{l}\text { I de } \\
\text { escolaridad }\end{array}$ & antidad & rcentaje \\
\hline 25- & 6 & 32. & Sin & 7 & 4 \\
\hline 35 años & 27 & $9 \%$ & escolaridad & 5 & $\%$ \\
\hline
\end{tabular}

Tabla 2

Nivel de escolaridad alcanzado por los padres y madres
Resultados
Datos biográficos
El instrumento en su mayoría fue contestado por las madres de familia con un $71.6 \%$, los padres contestaron en un $28.2 \%$, y solo un $0.2 \%$ (cuatro familias) fue contestado por ambos jefes de familia. Sobre la edad y escolaridad de los padres (Tabla 1 y 2), esta fluctúa entre los 36 y 50 años, y acreditaron solo el nivel básico de su educación formal.

Chontalpa en Tabasco, incluyendo los cinco municipios que la conforman; Cárdenas, Comalcalco, Cunduacán, Huimanguillo y Paraíso. Las familias que participan en esta exploración provienen de diferentes modelos familiares, características biográficas, económicas y sociales. 


\begin{tabular}{|c|c|c|c|c|c|c|c|}
\hline 36- & & 8 & 46. & Edu & & 1 & 59 \\
\hline 50 años & 79 & & $1 \%$ & cación básica & 131 & & $4 \%$ \\
\hline 51- & & 3 & 20. & Edu & & 4 & 23 \\
\hline 70 años & 99 & & $9 \%$ & cación media & 42 & & $2 \%$ \\
\hline $\sin$ & & 0 & $0 \%$ & Edu & & 2 & 13 \\
\hline respuesta & & & & $\begin{array}{c}\text { cación } \\
\text { superior }\end{array}$ & 57 & & $5 \%$ \\
\hline
\end{tabular}

Los resultados muestran que el $63.7 \%$ de las familias son de tipo nuclear; ambos padres e hijos viven bajo el mismo techo, seguidas por un $20.1 \%$ de familias extensas; donde además de vivir padres e hijos se encuentran abuelos, tíos, etc., es decir cohabitan tres generaciones, la familia monoparental que representa el $8.2 \%$, se refiere a aquellas familias integradas por los hijos y solo una de las figuras parentales, finalmente, la familia reconformada con $3.9 \%$, tiene que ver con la reintegración de familias monoparentales, no todos los hijos serán consanguíneos de ambos padres (el $4.1 \%$ de los padres y madres restantes no han especificado su modelo familiar). El nivel socioeconómico para fines prácticos lo distinguimos en solo tres niveles; nivel alto $(2.7 \%)$, nivel medio $(79.6 \%)$ y nivel bajo $(17.7 \%)$, es decir casi el $80 \%$ de las familias se declara de clase media, lo que resulta coherente dado que la población pertenece a los núcleos urbanos de los municipios, y en su gran mayoría cuentan con todos los servicios $\mathrm{y}$ satisfactores que les brinda residir en zonas urbanas.

\section{Modelo parental}

Los resultados se configuran a través de tres dimensiones específicas, las cuales en su conjunto ofertan aquellos modelos parentales culturalmente más arraigados en las familias objetos del estudio.

La tabla 3 representa el uso de pautas de crianza negociadoras en un $31.8 \%$; por lo que las normas y limites se manifiestan de acuerdo con la necesidad de los hijos, seguidos por aquellos padres rígidos 29.5\%; donde el énfasis en el orden y limites se manifiesta al 
Periódico do Núcleo de Estudos e Pesquisas sobre Gênero e Direito

Centro de Ciências Jurídicas - Universidade Federal da Paraíba

V. 9 - $\mathrm{N}^{\circ} 01$ - Ano 2020 - Spanish Edition

ISSN | 2179-7137 | http://periodicos.ufpb.br/ojs2/index.php/ged/index privilegiar la obediencia y el respeto a la autoridad. En esta caracterización de la pauta de crianza, se identifica además que aquellos que practican pautas de crianza negociadoras fueron padres jóvenes (pronta presencia de los hijos en la vida de la pareja), con un nivel socioeconómico medio y bajo, conforman una familia nuclear $\mathrm{o}$

Tabla 3

Caracterización del modelo parental respecto a las normas y disciplina

\begin{tabular}{lrr}
\hline \multicolumn{1}{c}{$\begin{array}{c}\text { Pauta } \\
\text { S de crianza }\end{array}$} & Porce \\
& & \\
ntaje & \\
iadora & Negoc & $31.8 \%$ \\
& Rígid & $29.5 \%$ \\
a & & \\
& Indul & $19.8 \%$ \\
gente & & $18.9 \%$ \\
& Situa & \\
cional & \multicolumn{2}{c}{ propia } \\
\hline
\end{tabular}

monoparental, con poco nivel educativo y con hijos menores de 18 años. A diferencia de los padres que practican pautas de crianza rígidas, los cuales pertenecen a familias de un nivel socioeconómico más elevado, cuya edad fluctúa entre 51 y 70 años, con nivel de educación básico o superior y con hijos comúnmente mayores de edad.
Tabla 4

Caracterización sobre

la demostración de afecto

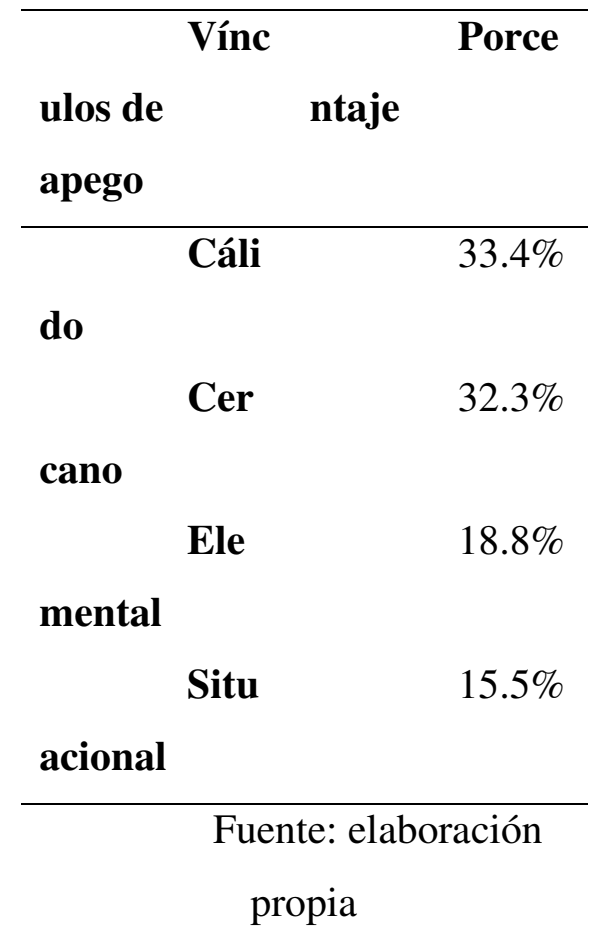


En la tabla 4 vemos que la cercanía en función del afecto y sus demostraciones se caracteriza por ser cálido y cercano, por lo que en términos generales el $65 \%$ de quienes participaron en esta exploración manifiesta el afecto hacia sus hijos de manera suficiente como para que los menores sean receptores de la aceptación y valor que les otorgan sus padres. Además de que el vínculo de apego cálido y cercano es el más manifestado en la dinámica familiar, también se denotan algunas características de los padres que pueden promover esta clase de interacción afectiva cercana y cálida en sus hogares; los padres con vínculos cercanos e hijos mayores de 18 años acreditaron una educación de media a media superior, conforman familias nucleares, monoparentales o reconformadas y comúnmente fueron padres jóvenes, cuya edad fluctúa a rededor de los 50 años, mientras que los padres con vínculos cercanos y con hijos menores de 18 años, registran poca escolaridad y su rango de edad fluctúa entre los 51 y 70 años.

\section{Tabla 5}

Caracterización de la expectativa y promoción del éxito

\begin{tabular}{lrr}
\hline \multicolumn{2}{c}{ Patrones } & Porcen \\
de éxito & taje \\
\hline r & Promoto & $26.4 \%$ \\
& Emancip & $25.2 \%$ \\
ador & & \\
& Orientad & $25.1 \%$ \\
or & & \\
& Situacion & $23.3 \%$ \\
al & & \\
\hline & & Fuente: elaboración propia
\end{tabular}


En coherencia con lo reportado en los vínculos de apego encontramos que los padres impulsan y orientan los intereses de sus hijos, promoviendo con ello un patrón promotor de éxito (tabla 5) que responde principalmente a sus propios intereses, necesidades $\mathrm{y}$ momento de desarrollo.

\section{Discusión}

Los resultados que se presentan hacen posible pensar en la estabilidad del modelo parental en diferentes espacios geográficos de México, donde las pautas de crianza negociadoras son seguidas por las rígidas, el vínculo de apego es cálido y cercano, y el patrón de éxito varía entre aquellos padres orientadores $\mathrm{y}$ promotores (Martínez et al., 2017).

$$
\text { Aquellos }
$$
hallazgos

relacionados con los componentes del modelo parental y los datos sociodemográficos de los padres contemplan una lógica cultural, por ejemplo, la relación entre calidez y edad de los hijos o la escolaridad de los padres, sin embargo, también es cierto que este tipo de relaciones están determinadas también por otras variables que intervienen en la cultura familiar, mismas que se han de contemplar en futuras exploraciones del modelo parental.

\section{Conclusiones}

Este estudio realizado en la zona de la Chontalpa del estado de Tabasco ha dejado ver como los modelos parentales en esta zona se configuran casi igual que en otras zonas del estado mexicano, exceptuando la diferencia que pude existir entre la promoción del éxito, pues los padres suelen ser más orientadores en el noreste de México, como se muestra en los resultados reportados por Martínez-Licona et al. (2016).

En esta zona el modelo "situacional" tanto de pautas de crianza, vínculos de apego y patrones de éxito, no se conforman de manera importante, lo que implica que los padres de la zona de la Chontalpa dentro de su cultura familiar conforman modelos parentales estables y coherentes a las características y cualidades de cada familia. Lo anterior se puede corroborar al evidenciar pautas de crianza negociadoras o rígidas, en términos generales apareciendo los condescendientes y situacionales no favorecidos de la misma manera, así mismo los vínculos de apego 
Periódico do Núcleo de Estudos e Pesquisas sobre Gênero e Direito

Centro de Ciências Jurídicas - Universidade Federal da Paraíba

V. 9 - $\mathrm{N}^{\mathrm{o}} 01$ - Ano 2020 - Spanish Edition

ISSN | 2179-7137 | http://periodicos.ufpb.br/ojs2/index.php/ged/index promovidos son más cálidos y cercanos, que situacionales y elementales, finalmente el fomento del éxito en la gran mayoría de las familias se mantiene como promotor más que orientador y emancipador.

\section{Referencias}

Caballero, T. y Castillo, M. (2003). Un análisis de la violencia dirigida hacia menores en el marco familiar. Vanvancito de 1997 al 2003. Universidad de Oriente. Santiago de Cuba. CUBA, 1-23.

Cadenas, H. y Urquiza, A. (2016). Naturaleza y contingencia en la Familia moderna. Espacio abierto, 25(3), 91100.

Cuervo, Á. (2010). Pautas de crianza y desarrollo socioafectivo en la infancia. Diversitas: Perspectivas en Psicología, $6(1), 111-121$.

Garcés, M. y Palacio, J. E. (2010). La comunicación familiar en asentamientos subnormales de montería (Colombia). Psicología desde el Caribe,1 (25), 1-29.
García, L. H. y Salazar, M. (2013). Crianza familiar en contextos margen de la ciudad de Cali: narrativas intergeneracionales. Ánfora, 20 (34), 3758.

González, J. C. (2011) Familia hoy: elementos para la discusión. Studiositas, 6(1), 53-70.

Martínez-Licona, J. F., Infante, A. y Medina, L. P. (2016). Ejes de racionalidad en torno a la familia y los modelos parentales: una construcción cualitativa de las teorías sobre la familia. En-claves del pensamiento, 10 (20), 115134.

Martínez, J. F., Díaz, A., Salazar, A. A. y Durón, M. (2014) Study of parental models: building an instrument for their exploration. Revista da Escola de Enfermagem da USP, 48(4), 594-601.

Martínez, J. F., García, M. I. y Collazo, J. C. (2017). Modelos parentales en el contexto urbano: un estudio exploratorio. Revista electrónica de investigación educativa, 19 (2), 54-66.

Martínez, J. F., Rodríguez, G. I., Diaz, A. y Reyes, M. A. (2018). Nociones y 
concepciones de parentalidad y familia.

Esc. Anna Nery, 20 (1), 1-9.

López, L. (2012). El cuidado de las hijas

y los hijos durante la migración internacional de los padres y las madres. Ánfora, 19 (32), 117-136.

Rodrigo, M. J., Máiquez, M. L. y Martín, J. C. (2010). Parentalidad positiva y políticas locales de apoyo a las familias. Federación española de municipios y provincias. Gobierno de España.

Rovira, M., Bernatzky, M. y Amores, F. (2009). Familias que hacen sociedad. Revista antiguos alumnos de IEEM, 12 (4), 78-83.

Solís, P. y Díaz, M. (2007). Relaciones entre creencias y prácticas de crianza de padres con niños pequeños. Anales de Psicología, 23 (2), 177-184.

Varela, S. P., Chinchilla, T. y Murad, V. (2015). Prácticas de crianza en niños y niñas menores de seis años en Colombia. Zona Próxima, 1(22), 193- 